\title{
Desarrollo y evaluación de la competencia transversal "pensamiento crítico" en el grado de ingeniería civil
}

\section{Víctor Yepes ${ }^{\mathrm{a}}$, José V. Martí y Tatiana García-Segura ${ }^{\mathrm{c}}$}

${ }^{a}$ ICITECH. Equipo de Innovación y Calidad Educativa EXCELCON. Departamento de Ingeniería de la Construcción y Proyectos de Ingeniería Civil. Universitat Politècnica de València, vyepesp@cst.upv.es, ${ }^{b}$ ICITECH. Equipo de Innovación y Calidad Educativa EXCELCON. Departamento de Ingeniería de la Construcción y Proyectos de Ingeniería Civil. Universitat Politècnica de València, jvmartia@cst.upv.es y ${ }^{\mathrm{c} I C I T E C H}$. Universitat Politècnica de València, tagarse@cam.upv.es

\begin{abstract}
This paper presents a methodology for assessing the cross disciplinary competence 'critical thinking' of the Civil Engineering undergraduate degree, in the subject of "Construction Methods II" of the 2nd year. An individual- and a group work based on the discussion of a construction method required for a bridge and its foundations is presented. This activity assesses both, the critical thinking competence on a rubric-based grading and the subject specific competences. A statistical-, a correlation analysis as well as multiple linear regressions of the group works and the individual exam marks were performed. Results show that nearly three-quarters of the students have achieved sufficient proficiency. However, results show a weak connection between the specific- and the cross-disciplinary competences. These evidences demonstrate that the acquisition of critical thinking competence is encouraged by the group work discussion. Nevertheless, the acquisition of specific competences requires not only a group work, but also an individual work.
\end{abstract}

Keywords: crossdisciplinar competences, critical thinking, civil engineering, undergraduate degree, multivariate analysis.

\footnotetext{
Resumen

La comunicación presenta una metodología para el desarrollo y evaluación de la competencia transversal "pensamiento crítico" en el grado de ingeniería civil, en el ámbito de la asignatura "Procedimientos de Construcción II" de segundo curso. Se presenta una actividad de trabajo individual y en grupo basada en la discusión del procedimiento constructivo de un puente y de sus cimentaciones. Dicha actividad permite la evaluación de la competencia de "pensamiento crítico" basada en una rúbrica, así como la evaluación de competencias específicas de la asignatura. Se ha realizado un análisis estadístico, de correlación y de regresión lineal múltiple de las calificaciones obtenidas en la actividad y en la prueba de evaluación contínua individual. Los resultados muestran como casi tres cuartas partes de los alumnos han alcanzado suficientemente la competencia. Sin embargo,
} 
Desarrollo y evaluación de la competencia transversal "pensamiento crítico" en el grado de ingeniería civil

los resultados muestran cierta desconexión entre los resultados relativos a las competencias específicas y los resultados relativos a la competencia transversal. Estas evidencias manifiestan que la adquisición de la competencia transversal del pensamiento crítico se ve favorecida por los trabajos de discusión en grupo. No obstante, la adquisición de competencias especificas por parte de los alumnos requiere no sólo de trabajos en grupo, sino también de trabajos individuales.

Palabras clave: competencias transversales, pensamiento crítico, ingeniería civil, grado, análisis multivariante

\section{Introducción}

La sociedad actual demanda nuevas competencias a los profesionales que requieren el dominio de destrezas y habilidades específicas (Vila y Poblete, 2007; Lee et al., 2013; Paslawski et al., 2015). La Escuela Técnica Superior de Ingenieros de Caminos, Canales y Puertos, siguiendo el objetivo estratégico de la Universitat Politècnica de València (UPV), está impulsando la evaluación de las competencias transversales, en particular en el grado de Ingeniería Civil. La asignatura de segundo curso y segundo cuatrimestre, Procedimientos de Construcción II, constituye un punto de control para evaluar la competencia transversal "Pensamiento Crítico", de la cual el primer autor es profesor responsable. Para el estudiante resulta muy importante acreditar su formación en competencias (http://www.enaee.eu/eurace-system), y para el empleador y la sociedad, resulta muy relevante conocer el nivel adquirido por el egresado (Ishengoma y Vaaland, 2016). Para la UPV supone una mejora continua de sus títulos universitarios, con un valor añadido innegable, facilitándose de esta forma la acreditación nacional e internacional de sus títulos (http://competencias.webs.upv.es/wp/).

El Equipo de Innovación y Calidad Educativa EXCELCON viene trabajando en los últimos años en el ámbito docente de la ingeniería y los proyectos de construcción (Pellicer et al., 2014). Así, Yepes et al. (2013) propusieron un sistema de indicadores para gestionar las competencias en los estudios de máster. Estos trabajos se complementaron muy recientemente con las aportaciones de Yepes et al. (2016). Pellicer et al. (2013) propusieron una metodología innovadora para planificar los estudios de posgrado en el ámbito de la gestión de la construcción que tuviese en cuenta la consecución de competencias. TorresMachí et al. (2013) introdujeron en estos trabajo el concepto de la empleabilidad de los graduados. Yepes (2014) estudia el resultado del uso del blog y las redes sociales en el aprendizaje activo en el ámbito de la construcción. El trabajo de Pellicer et al. (2016) propone una metodología de aprendizaje activo por parte de los alumnos para evaluar la sostenibilidad de las infraestructuras.

Atendiendo a la competencia transversal de pensamiento crítico, en trabajos anteriores (Martí y Yepes, 2015; Yepes y Martí, 2015) se realizó una aproximación previa a la valoración del pensamiento crítico como competencia transversal basada en la percepción de los alumnos respecto a su importancia, tanto en los grados de Ingenieros de Obras

(cc) EY-NC-ND 2016, Universitat Politècnica de València 
Públicas como los de Ingeniería Civil. Se trata de que los alumnos desarrollen un pensamiento crítico que se interese por los fundamentos en los que se asientan las ideas, acciones y juicios, tanto propios como ajenos.

\section{Objetivos}

Los objetivos de la comunicación son los siguientes:

1. Establecer una metodología a través de actividades grupales e individuales que permitan la evaluación de la competencia transversal "pensamiento crítico".

2. Conocer la relación existente entre el nivel de adquisición de la competencia "pensamiento crítico" y los resultados de aprendizaje de las competencias específicas de la asignatura.

3. Comprobar que es posible desarrollar actividades que permitan, de forma simultánea, la adquisición de competencias específicas y transversales.

\section{Desarrollo de la innovación}

La innovación planteada consiste en fundamentar el diseño de actividades basadas en metodologías activas de forma que se permita la evaluación de los resultados de aprendizaje más importantes de la competencia transversal "pensamiento crítico". Esta competencia implica cuestionar los supuestos subyacentes en la forma habitual de pensar y actuar y, partiendo de este pensamiento crítico, estar preparado para pensar y hacer de un modo diferente. El desarrollo y la evaluación de la competencia "pensamiento crítico" se basa en dos ejercicios que versan sobre la ejecución de la cimentación y la superestructura de un puente. Estas actividades se incluyen en un contexto de clase inversa, que ha empezado a utilizarse plenamente como metodología docente en esta asignatura durante el curso 201516. Estos ejercicios, además, permiten evaluar algunas de las competencias específicas de la asignatura como son:

a) Analizar críticamente los procesos propios de la Ingeniería Civil

b) Conocer y comprender las ciencias y las tecnologías correspondientes para la planificación, proyecto, construcción y explotación de las obrs propias del Sector de la Ingeniería Civil

c) Comprender los procedimientos constructivos, la maquinaria de construcción y las técnicas de organización, medición y valoración de obras.

Los alumnos previamente ya han realizado actividades sobre las unidades temáticas de construcción de cimentaciones y estructuras de contención, así como en la unidad de construcción de puentes. Para ello disponen de documentación preparada al efecto tanto en forma de blog (Yepes, 2016a) como de libro (Yepes, 2016b). Así, por ejemplo, los alumnos han presentado al resto de la clase la el procedimiento constructivo de un puente real, organizándose para ello en grupos de trabajo. Además, los dos ejercicios planteados forman parte del portafolio de 15 problemas prácticos que deben desarrollar los alumnos a lo largo del curso y que forman parte de la evaluación de la asignatura. Por tanto, los ejercicios

(cc)) BY-NC-ND 2016, Universitat Politècnica de València

Congreso In-Red (2016) 
Desarrollo y evaluación de la competencia transversal "pensamiento crítico" en el grado de ingeniería civil

planteados servirán tanto para la evaluación de la competencia transversal como para la evaluación de la asignatura. Los trabajos se realizan en grupo de un máximo de tres alumnos, con una parte individual que servirá para personalizar la evaluación de la competencia. Los grupos son los mismos que los que se han formado para el trabajo de presentación de puentes.

Los ejercicios están basados en sendos artículos de Millanes et al. (2014) y de GonzálezAranguren et al. (2014) donde las empresas IDEAM y SACYR explican, respectivamente, los detalles del proyecto y la construcción del viaducto de alta velocidad sobre el río Deba en la $\mathrm{Y}$ vasca (Figura 1). Se trata de una estructura de $900 \mathrm{~m}$ de longitud, con una altura máxima de pilas de $86 \mathrm{~m}$ y una distribución de luces de $50+80+70+60+3 \times 65+70+65+70+3 \times 65+45 \mathrm{~m}$, propiedad de Euskal Trenbide Sarea (ETS). La estructura salva un valle bastante profundo con una diferencia máxima de cota entre traza y terreno de unos $909 \mathrm{~m}$. La tipología estructural es de cajón de hormigón pretensado ejecutado con autocimbra, resaltando el récord de España de luz de tablero ejecutado con autocimbra en un viaducto de Alta Velocidad $(80 \mathrm{~m})$. Dada la gran altura del Viaducto y su localización en un valle muy visible, la solución para el viaducto del río Deba debía lograr conjugar la integración paisajística, minimizando las afecciones al entorno, compaginada con un proceso constructivo del tablero independiente del terreno. Los artículos mencionados se facilitan a los alumnos una vez han entregado los ejercicios resueltos, comprobando hasta qué punto coincide el planteamiento que han realizado con la realidad profesional. Ello también influye en su proceso de aprendizaje.

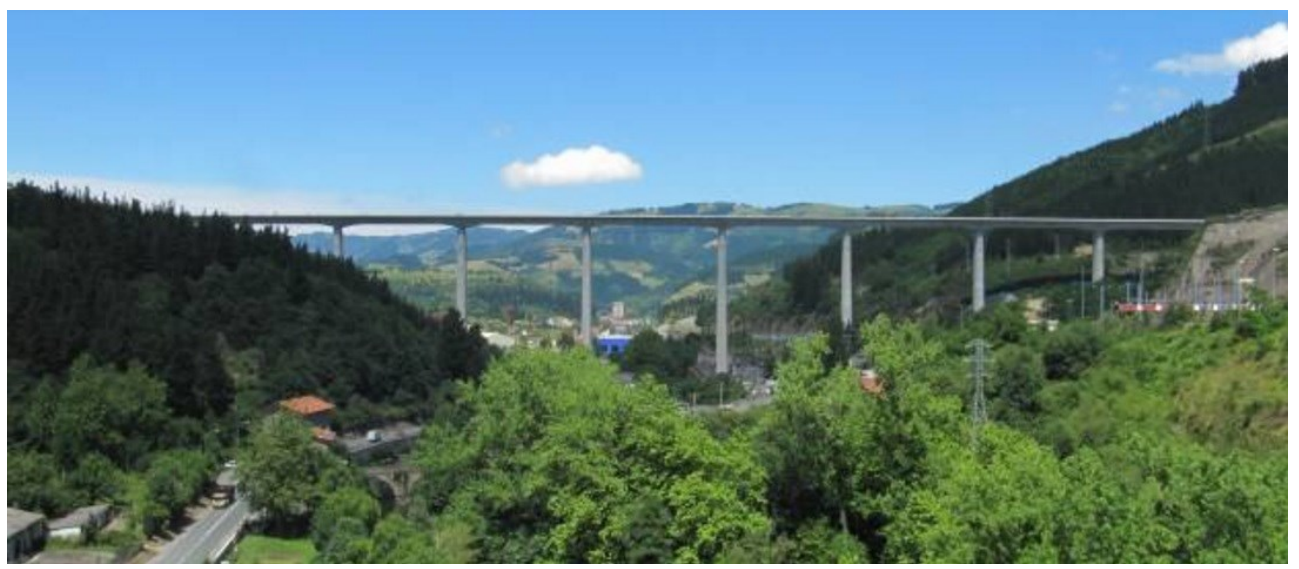

Fig. 1 Viaducto sobre el río Deba. Fuente: http://www.ideam.es/

En la Figura 2 se muestra el enunciado del problema real planteado. Conviene resaltar que algunos de los datos proporcionados son irrelevantes, otros son fundamentales y algún otro debe suponerse por parte de los alumnos de forma razonada. Este planteamiento permite ejercitar el pensamiento crítico respecto a la información disponible. En las Figuras 3 y 4 se muestran las preguntas a las que debe responder tanto el grupo como cada uno de los integrantes del grupo. A los alumnos también se les facilita, junto con los enunciados, la rúbrica con la cual van a ser evaluados (Figura 5). Los alumnos disponen de unas dos semanas para realizar completamente los ejercicios, que deberán entregarse antes de 
realizar la prueba individual de evaluación continua referida a las unidades temáticas correspondientes.

\section{ENUNCIADO}

Se quiere proyectar un viaducto para uso ferroviario de alta velocidad. Éste discurre sobre una vaguada por la que fluye un río con caudal muy variable. A $10 \mathrm{~km}$ se encuentra una ciudad con una población de 15000 habitantes Se trata de un valle profundo con una diferencia máxima de cota entre traza y terreno de más de $90 \mathrm{~m}$. El valle tiene una anchura de $900 \mathrm{~m}$ a la cota del tablero, y presenta un perfil en $\mathrm{V}$ bastante simétrico. El nuevo viaducto deberá respetar los cruces de carreteras y autopistas inferiores, además de la zona de servidumbre del río. Asimismo, la administración competente exige como medidas de impacto ambiental, que se respete el paso del aguilucho cenizo y que se minimice el riesgo de vertido de sustancias contaminantes sobre el río. El valle presenta una roca sana, que sólo en la zona adyacente al río, queda cubierta por un terreno de aluvial de $7 \mathrm{~m}$. Se esperan crecidas del río de hasta $3 \mathrm{~m}$ todos los años, siendo de hasta $10 \mathrm{~m}$ en casos excepcionales. La solución a proyectar debe conjugar la integración paisajística con el entorno, la facilidad constructiva, la viabilidad económica, así como los criterios de funcionalidad y estética adecuados. Use los datos del enunciado que considere importantes y, en el caso de necesitar datos, razone adecuadamente el uso de información adicional.

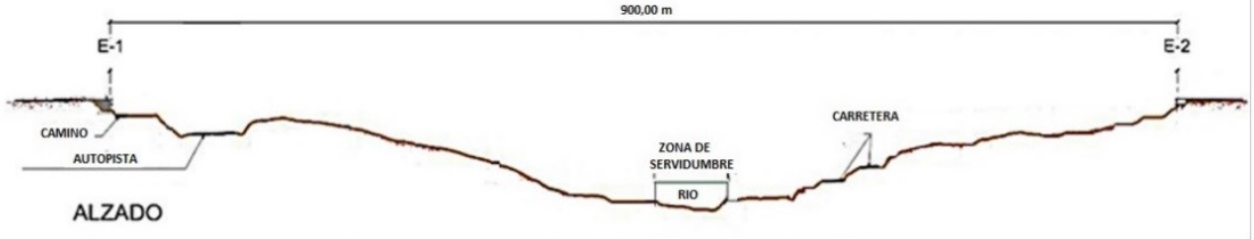

Fig. 2 Enunciado general de la actividad

\section{EJERCICIO 1}

\section{Preguntas de grupo}

1. Indique qué tipo de cimentación sería la más conveniente. No se exige que todas las cimentaciones sean iguales para todas las pilas o estribos; se adaptarán a cada caso.

2. Razone dos procesos constructivos que podrían ser aplicados y cuál de los dos cree que será más eficaz. La respuesta debe ser de consenso entre los miembros del grupo.

3. Define los principales pasos en la construcción de dichas cimentaciones.

4. Descarte, justificando las razones, al menos tres procesos constructivos de cimentación que no sean aplicables a este caso.

5. Indique si ha tenido que consultar otras fuentes para la elección de la tipología y el proceso constructivo (en dicho caso indicar cuál), o ha sido suficiente con el temario de la asignatura.

\section{Preguntas individuales}

a) Critique los dos procesos constructivos de la pregunta 2, indicando si está de acuerdo con lo consensuado por el grupo. Se valorará especialmente su opinión crítica personal justificada y si hay diversidad de opiniones entre los miembros del grupo.

b) Realice una crítica sobre el ejercicio 1 , indicando aquellas cosas con las que está de acuerdo con el grupo o no. Se valorará la justificación crítica de la respuesta.

Fig. 3 Preguntas relativas al Ejercicio 1

(cc) EY-NC-ND 2016, Universitat Politècnica de València

Congreso In-Red (2016) 
Desarrollo y evaluación de la competencia transversal "pensamiento crítico" en el grado de ingeniería civil

\section{EJERCICIO 2}

Preguntas de grupo

1. Indique qué tipología es la más indicada, así como la distribución de luces. Dibuje la solución propuesta sobre la figura anterior

2. Explique el razonamiento que se ha llevado a cabo para llegar a dicha solución.

3. Razone el proceso constructivo más conveniente para la ejecución del tablero.

4. Analice los pros y los contras de dicha solución. ¿Qué criterio ha sido el más relevante en la elección? ¿Presenta gran influencia la elección de la tipología y el proceso constructivo?

5. Indique si ha tenido que consultar otras fuentes para la elección de la tipología y el proceso constructivo (en dicho caso indicar cuál), o ha sido suficiente con el temario de la asignatura.

\section{Preguntas individuales}

a) Critique justificadamente su acuerdo con la solución a la que ha llegado el grupo en la pregunta 2. En caso de que los miembros del grupo propongan soluciones distintas, enumere las propuestas e indique si la toma de decisión ha sido por consenso. Se valorará especialmente su pensamiento crítico respecto al trabajo realizado.

b) Realice una crítica sobre el ejercicio 1 , indicando aquellas cosas con las que está de acuerdo con el grupo o no. Se valorará la justificación crítica de la respuesta.

Fig. 4 Preguntas relativas al Ejercicio 2

La rúbrica utilizada en la evaluación se basa en la propuesta realizada por un grupo de profesores de la Universitat Politècnica de València para el ICE. Dicha rúbrica hace referencia al nivel de dominio I, que es el adecuado para el segundo curso de un grado universitario. Dicha rúbrica el resultado de aprendizaje por el cual el alumno debe mostrar una actitud crítica ante la realidad, siendo capaz de analizar y cuestionar información, resultados, conclusiones y otros puntos de vista. Para evaluar dicho resultado de aprendizaje a dicho nivel se utilizan los siguientes indicadores, que ayudan a centrar la atención en los aspectos que se han de analizar para detectar los avances en los alumnos:

- Mostrar una actitud crítica ante la realidad: se pregunta el porqué de las cosas.

- Detectar incoherencias o contradicciones en el discurso de otras personas, o en un texto.

- Diferencias hechos de opiniones, interpretaciones o valoraciones.

- Profundizar en un tema con lógica e imparcialidad, contrastando información en fuentes fiables.

Diremos que el alumno ha desarrollado su pensamiento crítico si es capaz de interrogarse sobre la realidad e interesarse por los fundamentos en los que se asientan las ideas, las acciones, las valoraciones y los juicios, tanto propios como ajenos.

(c) EY-NC-ND 2016, Universitat Politècnica de València 


\begin{tabular}{|c|c|c|c|c|}
\hline \multirow{2}{*}{ INDICADORES } & \multicolumn{4}{|c|}{ DESCRIPTORES } \\
\hline & D. No alcanzado & C. En desarrollo & B. Bien / adecuado & A. Excelente/ejemplar \\
\hline $\begin{array}{l}\text { Muestra una actitud } \\
\text { crítica ante la realidad: } \\
\text { se pregunta el porqué } \\
\text { de las cosas }\end{array}$ & $\begin{array}{c}\text { No manifiesta ningún tipo } \\
\text { de espiritu crítico: nunca } \\
\text { se cuestiona la situación o } \\
\text { la realidad en la que vive. } \\
\text { Asume como cierta } \\
\text { cualquier información } \\
\text { que recibe }\end{array}$ & $\begin{array}{l}\text { Se cuestiona ciertas } \\
\text { situaciones de la realidad } \\
\text { en la que vive. } \\
\text { Pero es incapaz de emitir } \\
\text { juicios y valoraciones } \\
\text { propias. Necesita la } \\
\text { ayuda de otros para } \\
\text { obtener respuestas }\end{array}$ & $\begin{array}{l}\text { Se pregunta el por qué } \\
\text { de las cosas e investiga } \\
\text { para conseguir respuestas } \\
\text { de forma autónoma. } \\
\text { Pero se deja influir al emitir } \\
\text { sus propios juicios y } \\
\text { valoraciones }\end{array}$ & $\begin{array}{l}\text { Reflexiona e investiga el } \\
\text { por qué de las cosas, y es } \\
\text { capaz de encontrar } \\
\text { respuestas y } \\
\text { argumentarlas } \\
\text { objetivamente }\end{array}$ \\
\hline $\begin{array}{l}\text { Detecta incoherencias } \\
\text { o contradicciones en el } \\
\text { discurso de otras } \\
\text { personas o en un texto. }\end{array}$ & $\begin{array}{l}\text { No es capaz de detectar } \\
\text { incoherencias o } \\
\text { contradicciones en un } \\
\text { discurso o texto }\end{array}$ & $\begin{array}{l}\text { Es capaz de detectar } \\
\text { algunas incoherencias } \\
\text { pero no sabe explicar el } \\
\text { porqué de las mismas. }\end{array}$ & $\begin{array}{l}\text { Detecta incoherencias y } \\
\text { contradicciones, y aporta } \\
\text { argumentos para } \\
\text { evidenciar las mismas. }\end{array}$ & $\begin{array}{l}\text { Detecta incoherencias y } \\
\text { contradicciones, aporta } \\
\text { argumentos, y reformula } \\
\text { coherentemente los } \\
\text { enunciados } \\
\text { contradictorios. }\end{array}$ \\
\hline $\begin{array}{l}\text { Diferencia hechos de } \\
\text { opiniones, } \\
\text { interpretaciones o } \\
\text { valoraciones }\end{array}$ & $\begin{array}{c}\text { Demuestra una actitud } \\
\text { poco reflexiva ante el } \\
\text { discurso de otras } \\
\text { personas. } \\
\text { No distingue hechos de } \\
\text { opiniones } \\
\end{array}$ & $\begin{array}{l}\text { Normalmente distingue } \\
\text { hechos de opiniones, } \\
\text { pero puede aceptar } \\
\text { juicios o decisiones } \\
\text { basados en opiniones }\end{array}$ & $\begin{array}{l}\text { Diferencia hechos de } \\
\text { opiniones, } \\
\text { interpretaciones o } \\
\text { valoraciones en las } \\
\text { argumentaciones de } \\
\text { otros }\end{array}$ & $\begin{array}{l}\text { Cuestiona juicios o } \\
\text { decisiones basadas en } \\
\text { opiniones, valoraciones, } \\
\text { etc. y detecta falacias y } \\
\text { ambigüedades }\end{array}$ \\
\hline $\begin{array}{l}\text { Profundiza en un tema } \\
\text { con lógica e } \\
\text { imparcialidad, } \\
\text { contrastando } \\
\text { información en fuentes } \\
\text { fiables }\end{array}$ & $\begin{array}{l}\text { No es capaz de } \\
\text { profundizar en un tema. } \\
\text { Recurre a una única } \\
\text { fuente y no contrasta la } \\
\text { información }\end{array}$ & $\begin{array}{l}\text { Recurre a diversas } \\
\text { fuentes, pero no verifica } \\
\text { la fiabilidad de las } \\
\text { mismas. }\end{array}$ & $\begin{array}{l}\text { Consulta diferentes } \\
\text { fuentes y contrasta la } \\
\text { información de las mismas } \\
\text { para verificar su fiabilidad }\end{array}$ & $\begin{array}{l}\text { Consulta fuentes fiables, } \\
\text { contrasta la información y } \\
\text { aporta su valoración } \\
\text { personal }\end{array}$ \\
\hline
\end{tabular}

Fig. 5 Rúbrica UPV CT-09 sobre pensamiento crítico, nivel de dominio I

\section{Resultados}

A continuación se discuten y analizan los resultados obtenidos tras la realización de la actividad y la evaluación de la competencia transversal. Se han recogido las calificaciones obtenidas por 91 alumnos, de un total de 103 matriculados. Sólo se han considerado aquellos alumnos que han realizado la actividad y se han presentado a la prueba escrita de evaluación continua. Hay que indicar que el 22,0\% de los alumnos obtuvieron una calificación A, de excelente; el 50,5\% una calificación $\mathrm{B}$, de adecuado y un 27,5\% una calificación C, en desarrollo (Figura 6). Todos los alumnos evaluados superaron los indicadores previstos, no obteniendo nadie la calificación $\mathrm{D}$, no alcanzado. A efectos estadísticos, se ha valorado el A con 4 puntos, el B con 3 puntos, el C con 2 puntos y el D con 0 puntos.

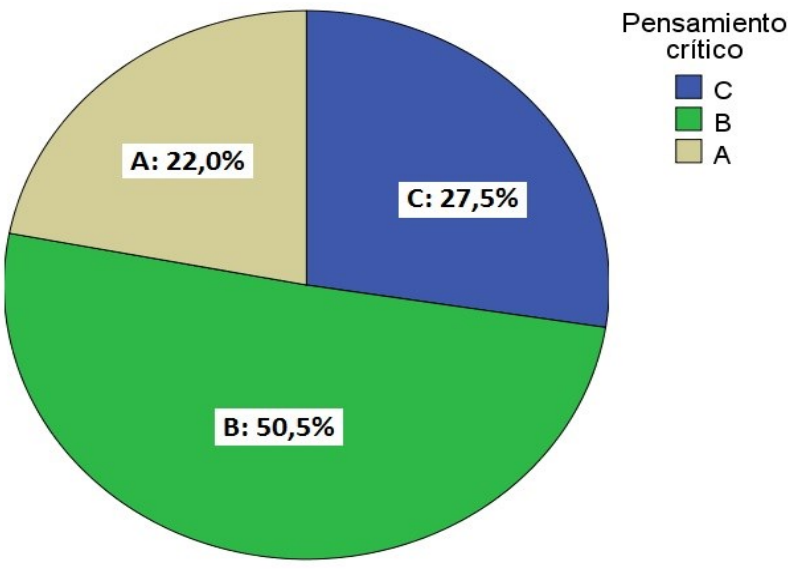

(c)) EY-NC-ND 2016, Universitat Politècnica de València

Congreso In-Red (2016) 
Desarrollo y evaluación de la competencia transversal "pensamiento crítico" en el grado de ingeniería civil

Fig. 6 Porcentajes de resultados en la evaluación de la competencia pensamiento crítico

En la Figura 7 y en la Tabla 1 se recogen los estadísticos descriptivos de las evaluaciones. Es importante destacar que las calificaciones de los Ejercicios 1 y 2 atienden exclusivamente al acierto técnico de las respuestas, mientras que la competencia transversal se centra en el desarrollo argumentativo y al pensamiento crítico empleado para llegar a los resultados. Los estadísticos de tendencia central (media, mediana y moda) de las calificaciones de los Ejercicios 1 y 2 son claramente superiores a los relativos a la calificación obtenida por los alumnos en la prueba escrita. Dicha desviación se puede explicar por la realización de los ejercicios en grupo, sin límite de tiempo y con la posibilidad de consultar las fuentes necesarias, frente a la evaluación individual realizada en la prueba escrita. Asimismo, en la Tabla 1 se refleja que las calificaciones de la prueba escrita presentan una mayor dispersión (desviación típica y rango) que el resto.

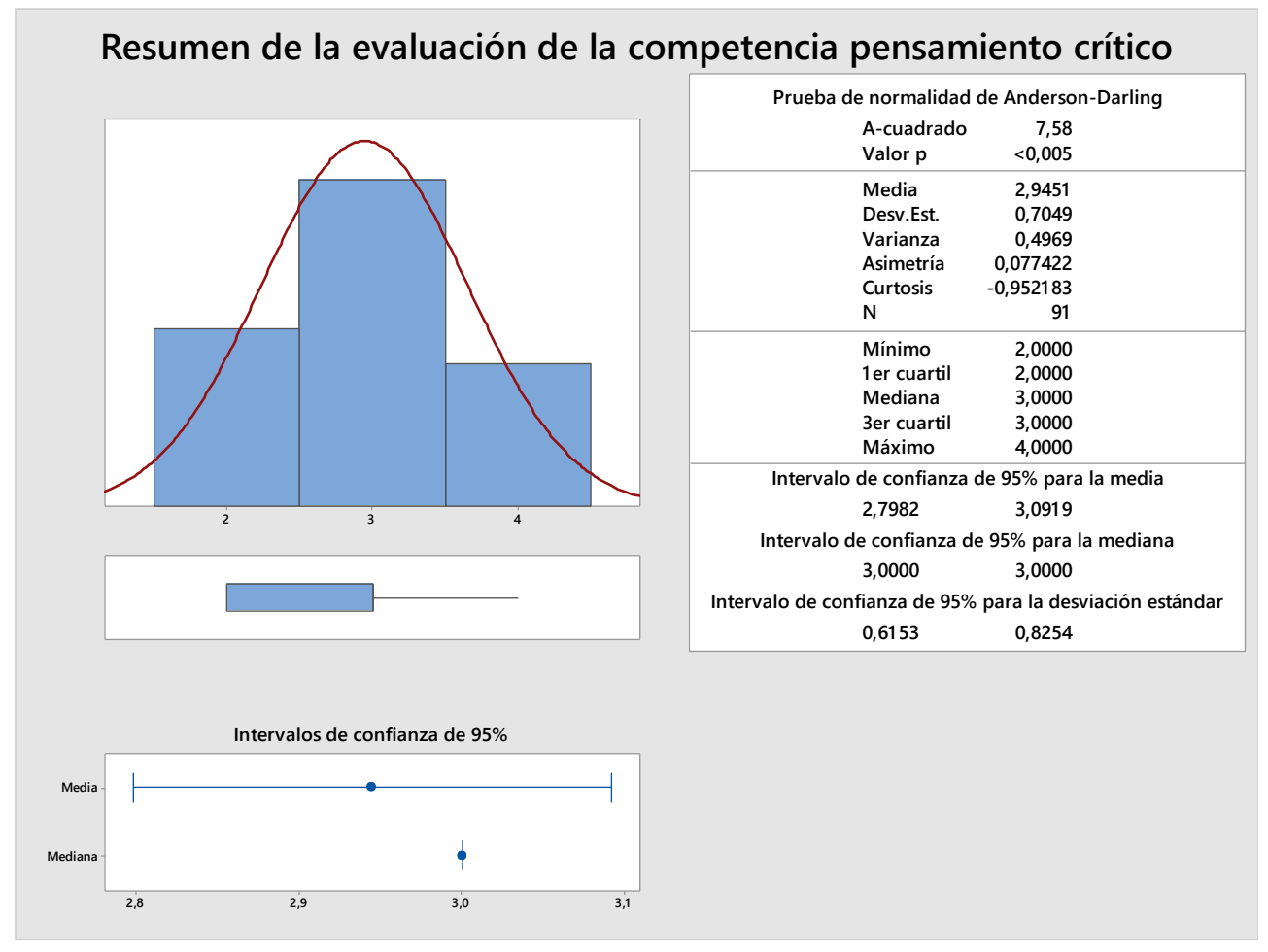

Fig. 7 Resumen estadísico de la evaluación de la competencia pensamiento crítico

Por otra parte, en la Figura 8 se muestra cómo las medias de las calificaciones de los ejercicios planteados no difieren estadísticamente entre sí, pero sí que lo hacen respecto a la calificación de la prueba escrita del examen, con un nivel de confianza del 95\%. Ello vuelve a confirmar la diferencia en los resultados entre los trabajos grupales e individuales.

(c)) EY-NC-ND 2016, Universitat Politècnica de València 
Tabla 1. Estadísticos descriptivos de las calificaciones

\begin{tabular}{lrrrrr}
\hline & & $\begin{array}{r}\text { Pensamiento } \\
\text { crítico }\end{array}$ & $\begin{array}{r}\text { Calificación } \\
\text { Ejercicio 1 }\end{array}$ & $\begin{array}{r}\text { Calificación } \\
\text { Ejercicio 2 }\end{array}$ & $\begin{array}{r}\text { Prueba } \\
\text { escrita }\end{array}$ \\
\hline N & Válidos & 91 & 91 & 91 & 91 \\
& Perdidos & 0 & 0 & 0 & 0 \\
\hline Media & 2,95 & 6,945 & 7,319 & 5,760 \\
Error típ. de la media &, 074 &, 2002 &, 2020 &, 2511 \\
Mediana & 3,00 & 7,000 & 7,500 & 5,700 \\
Moda & 3 & 9,5 & 9,0 & 5,5 \\
Desv. típ. &, 705 & 1,9097 & 1,9272 & 2,3956 \\
Rango & 2 & 6,0 & 7,0 & 9,9 \\
Mínimo & 2 & 3,5 & 3,0 &, 1 \\
Máximo & & 4 & 9,5 & 10,0 & 10,0 \\
\hline \multirow{2}{*}{ Percentiles } & 25 & 2,00 & 5,500 & 6,000 & 4,000 \\
& 50 & 3,00 & 7,000 & 7,500 & 5,700 \\
& 75 & 3,00 & 8,500 & 9,000 & 7,500 \\
\hline
\end{tabular}

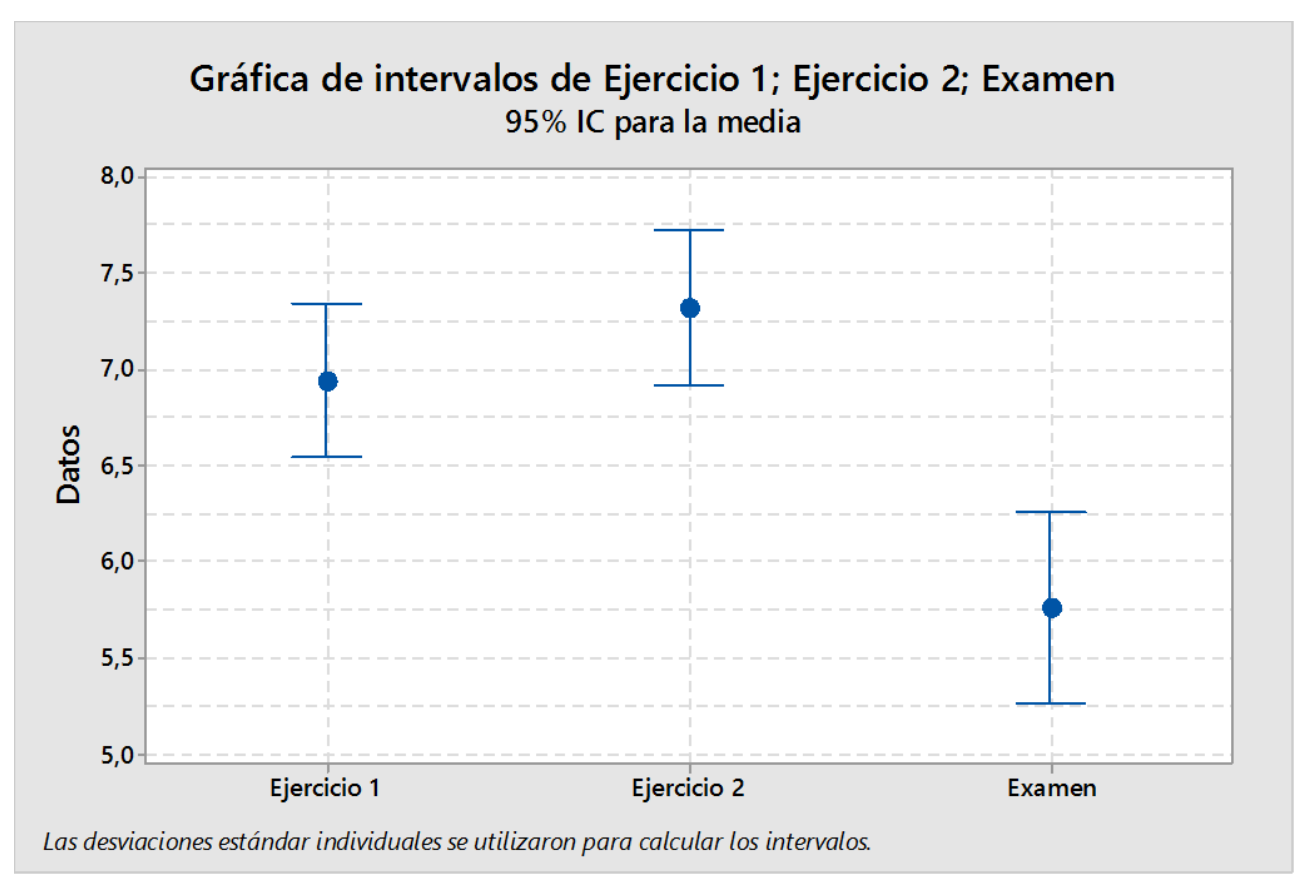

Fig. 8 Intervalos de confianza al 95\% para las medias de las calificaciones

2016, Universitat Politècnica de València 
Desarrollo y evaluación de la competencia transversal "pensamiento crítico" en el grado de ingeniería civil

Otro aspecto de gran interés es comprobar si exiten correlaciones significativas entre las calificaciones obtenidas por los ejercicios, la prueba escrita y la evaluación de la competencia transversal. En la Tabla 2 se muestran las correlaciones entre estas variables. Se comprueba que la evaluación de la competencia transversal es significativa al nivel 0,01 (bilateral), aunque con un coeficiente de correlación de Pearson moderado, de al menos 0,639. Sin embargo, la correlación entre los resultados del examen escrito y los ejercicios, aunque significativa, presenta un coeficiente bajo, que no supera el 0,439. La correlación entre variables puede visualizarse en las gráficas de dispersión mostradas en la Figura 9. Se comprueba cierto alineamiento de puntos entre las calificaciones de los Ejercicios 1 y 2 , que contrasta con la nube de puntos existente cuando se comparan dichas calificaciones con la de la prueba escrita.

Tabla 2. Correlaciones entre las distintas calificaciones

\begin{tabular}{|c|c|c|c|c|c|}
\hline & & $\begin{array}{c}\text { Pensamiento } \\
\text { crítico } \\
\end{array}$ & $\begin{array}{c}\text { Calificación } \\
\text { Ejercicio } 1 \\
\end{array}$ & $\begin{array}{c}\text { Calificación } \\
\text { Ejercicio } 2 \\
\end{array}$ & $\begin{array}{c}\text { Calificación } \\
\text { Examen } \\
\end{array}$ \\
\hline \multirow[t]{3}{*}{ Pensamiento crítico } & Corr. de Pearson & 1,000 &, $782^{* *}$ &, $639^{* *}$ &, $671^{* *}$ \\
\hline & Sig. (bilateral) & &, 000 &, 000 &, 000 \\
\hline & $\mathrm{N}$ & 91 & 91 & 91 & 91 \\
\hline Calificación & Corr. de Pearson &, $782^{* *}$ & 1,000 &, $671^{* *}$ &, $439^{* *}$ \\
\hline \multirow[t]{2}{*}{ Ejercicio 1} & Sig. (bilateral) &, 000 & &, 000 & ,000 \\
\hline & $\mathrm{N}$ & 91 & 91 & 91 & 91 \\
\hline Calificación & Corr. de Pearson &, $639^{* *}$ &, $671^{* *}$ & 1,000 &, $293^{* *}$ \\
\hline \multirow[t]{2}{*}{ Ejercicio 2} & Sig. (bilateral) &, 000 &, 000 & &, 005 \\
\hline & $\mathrm{N}$ & 91 & 91 & 91 & 91 \\
\hline Calificación & Corr. de Pearson &, $671^{* *}$ &, $439^{* *}$ &, $293^{* *}$ & 1,000 \\
\hline \multirow[t]{2}{*}{ Examen } & Sig. (bilateral) &, 000 &, 000 &, 005 & \\
\hline & $\mathrm{N}$ & 91 & 91 & 91 & 91 \\
\hline
\end{tabular}

**. La correlación es significativa al nivel 0,01 (bilateral). 


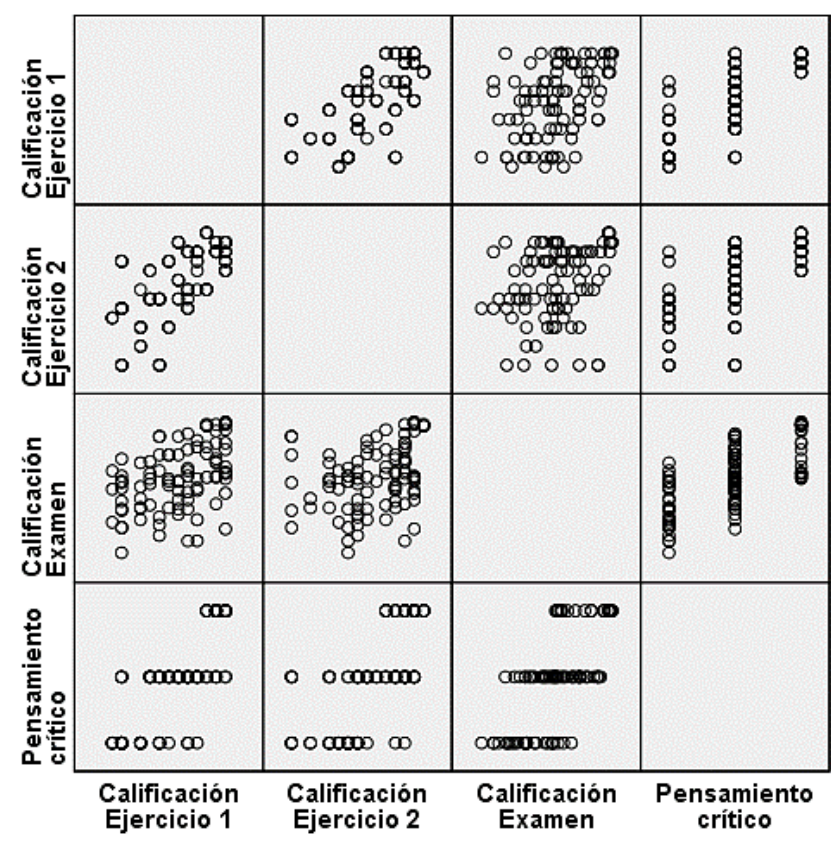

Fig. 9 Gráficas de dispersión entre las variables

A continuación realizamos un análisis de regresión de todas las variables para intentar establecer modelos que expliquen la variable dependiente que elijamos. Para ello se realizan inferencias acerca de modelos lineales simples o múltiples y se obtienen medidas cuantitativas del grado de relación de las variables a través del coeficiente de correlación $R$. Los modelos lineales se ajustan por mínimos cuadrados de forma que la variable dependiente se encuentre explicada lo máximo posible por un conjunto de variables independientes. La bondad del ajuste se evalúa mediante el coeficiente de determinación $R^{2}$, que se interpreta como la proporción de variación de la variable de respuesta explicada mediante el modelo de regresión lineal (Draper y Smith, 1999).

En primer lugar, se intenta explicar cada variable de respuesta en función de aquella variable independiente con la cual se encuentra más correlacionada. Se trata de aumentar el coeficiente de regresión incorporando variables independientes explicativas. Para ello se procede mediante el método stepwise de pasos sucesivos (Hocking, 1976), consistente en introducir las variables una por una y comprobar si la variable permanece o sale del modelo. Se toma como criterio de inclusión un incremento en la varianza explicada significativo al $5 \%(F=0,050)$, mientras que para excluir una variable se considera un decremento del $10 \%(F=0,100)$. La primera variable introducida es la que presenta un coeficiente de correlación $R$ más alto. A continuación se vuelven a calcular todas las correlaciones eliminando la influencia de aquella que ya ha entrado en el modelo, y se introduce la siguiente con mayor $R$; de esta forma se consigue que las variables que entren no sean dependientes de las que ya figuran en el modelo. 
Desarrollo y evaluación de la competencia transversal "pensamiento crítico" en el grado de ingeniería civil

Como resultado de la regresión múltiple realizada (Tablas 3 y 4) se comprueba que la calificación obtenida al evaluar la competencia transversal pensamiento crítico se puede explicar con las calificaciones de los ejercicios y de la prueba escrita. De hecho, en la Tabla 3 se muestran tres modelos lineales en función de las variables predictoras. La calificación del Ejercicio 1 explica por sí sola el 60,7\% de la variabilidad observada en la evaluación de la competencia transversal. Hay que introducir la calificación del examen y del Ejercicio 2 para explicar el $76,1 \%$ de la variabilidad. En la Tabla 4 se detallan los coeficientes de los modelos lineales resultantes.

Tabla 3. Resumen del modelo de regresión lineal

\begin{tabular}{ccccc}
\hline Modelo & R & R cuadrado & $\begin{array}{c}\text { R cuadrado } \\
\text { corregida }\end{array}$ & Error típ. de la estimación \\
\hline 1 &, $782^{\mathrm{a}}$ &, 611 &, 607 &, 442 \\
2 &, $863^{\mathrm{b}}$ &, 745 &, 739 &, 360 \\
3 &, $877^{\mathrm{c}}$ &, 769 &, 761 &, 345 \\
\hline
\end{tabular}

a. Variables predictoras: (Constante), Calificación Ejercicio 1

b. Variables predictoras: (Constante), Calificación Ejercicio 1, Calificación Examen

c. Variables predictoras: (Constante), Calificación Ejercicio 1, Calificación Examen, Calificación

Ejercicio 2

Tabla 4. Coeficientes de los modelos de regresión lineal

\begin{tabular}{|c|c|c|c|c|c|c|}
\hline & & \multicolumn{2}{|c|}{$\begin{array}{l}\text { Coeficientes no } \\
\text { estandarizados }\end{array}$} & \multirow{2}{*}{$\begin{array}{c}\text { Coeficientes } \\
\text { estandarizados } \\
\text { Beta } \\
\end{array}$} & \multirow[b]{2}{*}{$\mathbf{t}$} & \multirow[b]{2}{*}{ Sig. } \\
\hline \multicolumn{2}{|c|}{ Modelo } & B & Error típ. & & & \\
\hline \multirow[t]{2}{*}{1} & (Constante) & ,941 &, 176 & & 5,355 & ,000 \\
\hline & Calificación Ej. 1 & ,289 &, 024 & ,782 & 11,829 & ,000 \\
\hline \multirow[t]{3}{*}{2} & (Constante) & ,709 & ,147 & & 4,814 &, 000 \\
\hline & Calificación Ej. 1 & ,223 &, 022 & ,604 & 10,070 &, 000 \\
\hline & Calificación Examen &, 120 &, 018 & ,406 & 6,781 &, 000 \\
\hline \multirow[t]{4}{*}{3} & (Constante) & 509 & 156 & & 3,264 & ,002 \\
\hline & Calificación Ej. 1 &, 171 &, 027 &, 464 & 6,271 &, 000 \\
\hline & Calificación Examen &, 120 &, 017 & ,407 & 7,085 &, 000 \\
\hline & Calificación Ej. 2 & ,076 & 025 & ,208 & 2,999 & ,004 \\
\hline
\end{tabular}

a. Variable dependiente: Pensamiento crítico 


\section{Conclusiones}

La presente comunicación presenta la viabilidad de una metodología de desarrollo y evaluación de la competencia transversal "pensamiento crítico" basada en una actividad de trabajo en equipo, con una componente individual. La evaluación de la competencia se ha realizado mediante el empleo de una rúbrica que mide el resultado de aprendizaje de la competencia en el nivel de dominio adecuado al segundo curso de un grado universitario. Los resultados muestran como casi tres cuartas partes de los alumnos han alcanzado suficientemente la competencia. Sin embargo, los resultados muestran cierta desconexión entre los resultados académicos relativos a las competencias específicas evaluadas por una prueba individual escrita y los resultados relativos a la competencia transversal. Estas evidencias manifiestan que la adquisición de la competencia transversal del pensamiento crítico se ve favorecida por los trabajos de discusión en grupo, por la necesidad de llegar a acuerdos razonados entre los distintos miembros. Sin embargo, la adquisición de competencias específicas por parte de los alumnos requiere no sólo de trabajos en grupo, sino también de trabajos individuales. Otra conclusión importante del presente estudio es comprobar que una actividad que permite evaluar la adquisición de competencias específicas, también puede servir para la evaluación de una competencia transversal.

\section{Agradecimientos}

Los autores agradecen el apoyo recibido por el Ministerio de Economía y Competitividad y de los fondos FEDER (Proyecto de Investigación BIA2014-56574-R) y por la Universitat Politècnica de València (Equipo de Innovación y Calidad Educativa EXCELCON).

\section{Referencias}

DRAPER, N.; SMITH, H. (1999). Applied regression analysis. New York: Wiley.

ENAEE. The EUR-ACE® System <http://www.enaee.eu/eur-ace-system> [Consulta: 22 de marzo de 2016]

GONZÁLEZ-ARANGUREN, J.; REDERO, A.; IMEDIO, J. PÉREZ-FERRERUELA, J.; CAÑIZAL, J. (2014). Análisis constructivo y ejecución del viaducto de alta velocidad sobre el río Deba en la Y vasca. En: VI Congreso Internacional de Estructuras ACHE. 3-5 de junio, Madrid, pp. 1-10.

HOCKING, R. (1976). "The analysis and selection of variables in linear regression" en Biometrics, vol. 32, p. 1-49.

ISHENGOMA, E.; VAALAND, T.I. (2016). "Can university-industry linkages stimulate student employability?", en Education and Training, vol. 58, issue 1, p. 18-44.

LEE, S.; AFSHIN, E.; LEE, D.E. (2013). "Graduate construction management programs in the US: Lessons learned from leading institutions", en KSCE Journal of Civil Engineering, vol. 17, issue 7, p. 1664-1671.

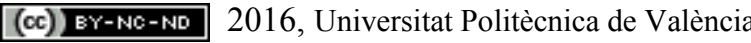

Congreso In-Red (2016) 
MARTÍ, J.V.; YEPES, V. (2015). "Pensamiento crítico como competencia transversal en el grado de Ingeniería de Obras Públicas: valoración previa“. En: Congreso Nacional de Innovación Educativa y Docencia en Red In-Red 2015, 30 de junio - 1 de julio, Universitat Politècncia de València, pp. 1-12.

MillanES, F.; ORTEGA, M.; SOLERA, P.; FIGUEIREDO, H.; UGARTE, J. (2014). Concepción y proyecto del viaducto de alta velocidad sobre el río Deba en la Y vasca. En: VI Congreso Internacional de Estructuras ACHE. 3-5 de junio, Madrid, pp. 1-10.

PASLAWSKI, J.; MILWICZ, R.; NOWOTARSKI, P. (2015). "Modernization of curriculum in construction management based on EU funds", en Archives of Civil Engineering, vol. 61, issue 4, p. 175-186.

PELLICER, E.; SIERRA, L.A.; YEPES, V. (2016). "Appraisal of infrastructure sustainability by graduate students using an active-learning method", en Journal of Cleaner Production, vol. 113, p. 884-896.

PELLICER, E.; YEPES, V.; ORTEGA, A.J. (2013). "Method for planning a graduate program in construction management", en Journal of Professional Issues in Engineering Education and Practice ASCE, vol 139, issue 1,p. 33-41.

PELliCER, E.; YEPES, V.; TEIXEIRA, J.C.; MOURA, H.P.; CATALÁ, J. (2014). Construction Management. New York: Wiley Blackwell.

TORRES-MACHÍ, C.; CARRIÓN, A.; YEPES, V.; PELLICER, E. (2013). "Employability of graduate students in construction management", en Journal of Professional Issues in Engineering Education and Practice ASCE, vol. 139, issue 2, p. 163-170.

UNIVERSITAT POLITĖCNICA DE VALÉNCIA. Competencias transversales $<\mathrm{http}$ ://competencias.webs.upv.es/wp/> [Consulta: 22 de marzo de 2016]

VILA, A.; POBLETE, M. (2007). Aprendizaje basado en competencias. Una propuesta para la evaluación de las competencias genéricas. Ediciones Mensajero, Bilbao.

YEPES, V. (2014). "El uso del blog y las redes sociales en la asignatura de Procedimientos de Construcción". En: Jornadas de Innovación Educativa y Docencia en Red IN-RED 2014. 15-16 de julio, Valencia, pp. 1-9.

YEPES, V. (2016a). "Procedimientos de construcción de cimentaciones y estructuras de contención". Colección Manual de Referencia. Editorial Universitat Politècnica de València, $202 \mathrm{pp}$.

YEPES, V. (2016b). Procedimientos de Construcción. < http://procedimientosconstruccion.blogs.upv.es> [Consulta: 22 de marzo de 2016]

YEPES, V.; MARTÍ, J.V. (2015). “Competencia transversal 'pensamiento crítico' en el grado de ingeniería civil: valoración previa“. En: XIII Jornadas de Redes de Investigación en Docencia Universitaria. 2-3 de julio, Alicante, pp. 2944-2952.

YEPES, V.; PELLICER, E.; ORTEGA, J.A. (2012). "Designing a benchmark indicator for managerial competences in construction at the graduate level", en Journal of Professional Issues in Engineering Education and Practice ASCE, vol. 138, issue 1, p. 48-54.

YEPES, V.; SEGADO, S.; PELLICER, E.; TORRES-MACHÍ, C. (2016). "Acquisition of competences in a Master Degree in Construction Management". En: 10th International Technology, Education and Development Conference (INTED 2016). 7-9 de marzo, Valencia, pp. 718-727. 